EPJ Web of Conferences 81, 01004 (2014)

DOI: $10.1051 /$ epjconf/ 20148101004

(C) Owned by the authors, published by EDP Sciences, 2014

\title{
New insights on spectroscopy and properties of light hadrons with COMPASS
}

\author{
Florian Haas $^{1, a}$ \\ for the COMPASS Collaboration \\ ${ }^{1}$ Physik Department E18, Technische Universität München
}

\begin{abstract}
COMPASS is a multi-purpose fixed-target experiment at the CERN Super Proton Synchrotron aimed at studying the structure and spectrum of hadrons. One main goal is the search for new hadronic states, in particular hybrid mesons and glueballs. Its large acceptance, high resolution, and high-rate capability make the COMPASS experiment an excellent device to study the spectrum of light-quark mesons in diffractive and central production up to masses of about $2.5 \mathrm{GeV} / \mathrm{c}^{2}$. COMPASS is able to measure final states with charged as well as neutral particles, so that resonances can be studied in different reactions and decay channels. During 2008 and 2009, COMPASS took a large data sample using $190 \mathrm{GeV} / c$ negative and positive hadron beams on various targets. We present new results from the analyses of this data set. One focus lies on the search for new mesons in diffractively produced multi-particle final states. Here novel analysis methods are applied to study the dependence of partial waves on the squared four-momentum transfer $t^{\prime}$ from the beam to the target. This also leads to a better separation of resonant and nonresonant contributions. Finally an update will be given on the recent analysis of the pion polarizability, which tests chiral dynamics.
\end{abstract}

\section{Motivation}

The development of Quantum Chromodynamics (QCD) brought up new features which had been never treated in any field theory before. Besides the interaction of quark and gluon fields, the selfcoupling of gluons has to be considered. This results on the one hand in the assymptotic freedom with quarks and gluons as the relevant degrees of freedom. In this regime the strong coupling constant is small, so that the number of loop corrections is reduced to a small number and perturbative calculations can be applied. On the other hand, in the field of confinement, hadrons are the relevant degrees of freedom and pQCD methods are not applicable. Concepts like the quarkmodel and effective theories as the chiral perturbation theory are used to describe light hadrons. Within the quark model, mesons are described as bound quark-antiquark pairs, neglecting any gluonic content. Thus not all spin-parity states can be realised based on this assumptions. More advanced QCD-inspired models allow for spin-exotic quantum numbers, e.g. $J^{P C}=1^{-+}$, by taking excited gluonic field configurations between the two quarks into account. The experimental observation of such a spin-exotic state would proove the existence of states beyond the well-established quark model. Apart from that topic

\footnotetext{
ae-mail: florian.haas@tum.de
} 
an experimental investigation of the light hadronic spectrum is still neccessary and new obseravtions can sheed light on this kinematical regime.

\subsection{The COMPASS experiment}

The multi-purpose fixed-target experiment COMPASS [1] can contribute significantly to the study with light hadrons. It is located at the SPS accelerator at CERN at the M2 beamline, providing the spectrometer various kinds of hadron beams, like secondary pion and kaon beams or tertiary muon beams in a momentum range between 160 to $220 \mathrm{GeV} / c$. The acceptance of the two-stage magnetic spectrometer covers a large kinematic realm of the outgoing particles. Both parts of the experiment are equipped with tracking and calorimetry which allows for the precise detection of charged as well as neutral particles. In addition a ring-imaging Cherenkov counter for PID is installed in the first magnetic stage. For the hadron structure and spectroscopy data taking campaign in 2008 and 2009, a $40 \mathrm{~cm}$ long liquid-hydrogen target surrounded by a recoil-proton detector (RPD) was used. The RPD was acting as the main trigger component and a silicon beam-telescope was placed upstream the target.

\section{Light meson spectroscopy}

The hadron structure and spectroscopy physics program of COMPASS covers a variety of physics processes and corresponding analyses, such as baryon spectroscopy, possible violations of the OZI rule [2] or the study of processes in the overlap region of strong and electromagnetic interactions [3]. A major topic is light-meson spectroscopy with the focus on central production and diffractively produced systems, like the $\eta \pi$ and $\eta^{\prime} \pi$ final states [4] or the three-pion systems, which will be discussed in more detail in the following sections.

\subsection{Partial-wave analysis}

The basic concepts of the Partial-Wave Analysis (PWA) applied to the COMPASS data will be explained by means of the diffractively produced three-pion systems. In this process the incoming negatively charged beam pion, with a beam momentum of $190 \mathrm{GeV} / c$, gets excited, via Pomeron exchange with the target proton, to an intermediate state $X^{-}$which then decays into the final state. The $X^{-}$cannot be measured directly and thus its corresponding quantum numbers $J^{P C} M^{\varepsilon}$, with $J$ the spin, $P$ and $C$ the eigenvalues of parity and charge conjugation, $M$ the spin projection and $\varepsilon$ the reflectivity of $X^{-}$, are unknown. Based on the underlaying physcis process of diffraction many combinations of these quantum numbers are possible and they may all interefere. Hence the aim of the PWA is to distentangle the various contributions of all these intermediate states.

In contrast to its production, the decay of $X^{-}$can be described since the final-state particles with corresponding phase-space variables $\tau$ are measured by COMPASS. The decay is parametrised within the isobar model, assuming that $X^{-}$first decays into a negative pion and an intermediate substate, the isobar, which finally decays into two pions (c.f. Fig. 1a). The applicability of the isobar model is illustrated by the Dalitz distribution in the $\pi_{2}(1670)$ mass region (c.f. Fig. 1b), which shows $\rho(770)$ and $f_{2}(1270)$ bands, the two major decay branches of the $\pi_{2}$. Finally the observed intensity $I(\tau)$ is given by:

$$
I\left(m_{X}, \tau\right)=\left|\sum_{\text {wave }} T_{\text {wave }}\left(m_{X}\right) \psi_{\text {wave }}(\tau)\right|^{2} .
$$




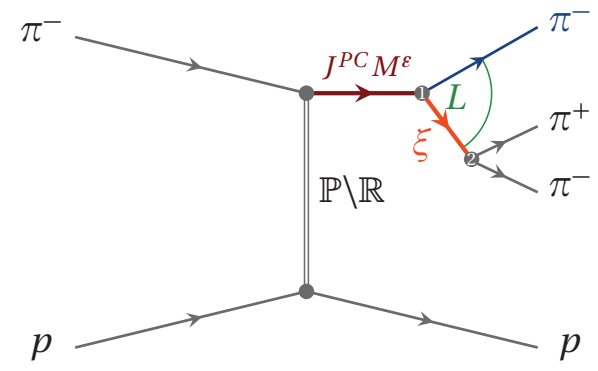

(a)

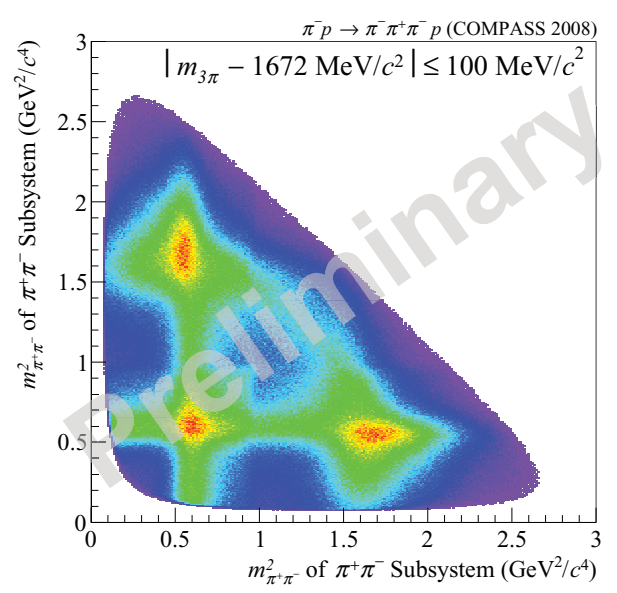

(b)

Figure 1: Schematic view of the $X^{-}$decay into the $3 \pi$ final state, described within the isobar model (a). Dalitz plot of the $\pi^{-} \pi^{+} \pi^{-}$final state in the mass region of the $\pi_{2}$ (1670) (b).

The unknown production amplitudes $T_{\text {wave, }}$, which have to be determined by the PWA, describe the production of the intermediate state $X^{-}$. The calculable decay amplitudes $\psi_{\text {wave }}$ depend on the phasespace variables $\tau$. The intensity in Eq. (1) is a coherent sum of partial waves, each given by:

$$
J^{P C} M^{\varepsilon}[\text { isobar }] \pi L,
$$

where $J^{P C} M^{\varepsilon}$ defines the intermediate state $X^{-}$and the decay is fixed by the isobar and the orbital angular momentum $L$ between the isobar and the spectator pion. The waveset of the three-pion analyses consists of 87 partial waves with spin $J$ and angular momentum $L$ up to six and with the isobars

$$
(\pi \pi)_{S}, f_{0}(980), f_{0}(1500), \rho(770), \rho_{3}(1630) \text { and } f_{2}(1270) .
$$

In addition an isotropic wave is added incoherently to the observed intensity.

\subsection{The $\pi^{-} \pi^{+} \pi^{-}$and $\pi^{-} \pi^{0} \pi^{0}$ final states}

For the charged three-pion channel, $\pi^{-} \pi^{+} \pi^{-}$, about 50 million events were accepted, the wolrd's largest data sample of this kind. For the neutral channel, $\pi^{-} \pi^{0} \pi^{0}$, about 3.5 million events were recorded. The kinematic range of interest is between 0.5 and $2.5 \mathrm{GeV} / c^{2}$ of the invariant three-pion mass and 0.1 and $1.0 \mathrm{GeV}^{2} / c^{2}$ for the squared four-momentum transfer $t^{\prime}$. The reconstruction of the two final states relies on different detectors so that a comparison of the two reveals systematic errors. Different PWA software packages were applied to the two sets. Since both final states only differs in the respective decay modes, the same contributions of intermediate states $X^{-}$are expected.

\subsubsection{A new axialvector resonance - the $a_{1}(1420)$}

Besides known resonances, found in the data, like e.g. $a_{1}(1260), a_{2}(1320)$ or $\pi_{2}(1670)$, COMPASS observes a striking peak structure in the $1^{++} 0^{+} f_{0}(980) \pi P$ wave, seen in both final states (c.f. Fig. 2a). 


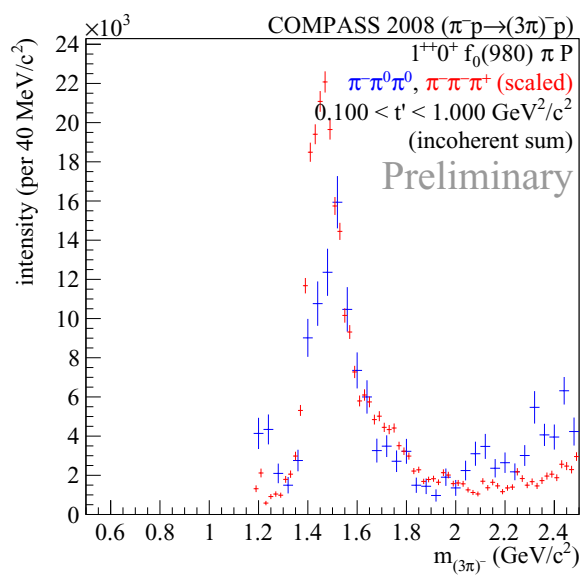

(a)

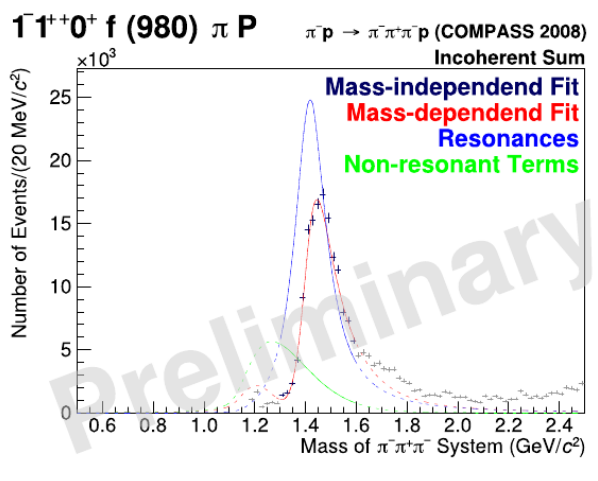

(b)

Figure 2: Intensity of the $1^{++} 0^{+} f_{0}(980) \pi P$ wave, seen in the charged channel (red) and the neutral one (blue) (a). $\chi^{2}$-fit of this wave in the charged channel, based on a six-wave spin-density submatrix (b).

The $a_{1}(1420)$ has the same quantum numbers like the biggest wave in the set, the $1^{++} 0^{+} \rho \pi S$, but differs in the decy channel. No structure was observed by previous expereminets in the $1^{++} 0^{+} f_{0}(980) \pi P$ wave. Phase motion between these wave with respect to other established waves indicates a resonant structure which could be confirmed by a $\chi^{2}$-fit on a spin-density sub-matrix (c.f. $2 b$ ), consisting of six partial waves. The resonance parameters of this $a_{1}(1420)$ were determined to be in the mass range of $1412-1422 \mathrm{MeV} / c^{2}$ and a width between $130-150 \mathrm{MeV} / c^{2}$. Not only its very small contribution to the overall intensity at the per-mille level, but also the fact that the $a_{1}(1420)$ decays only to the $f_{0}(980)$ isobar, which indicates a strong coupling to $K K$, make this observation peculiar. In addition the mass of the $a_{1}(1420)$ is close to the $K K^{*}$ threshold. Summarising the nature of this state is unclear for the moment.

\subsubsection{The spin-exotic $J^{P C}=1^{-+}$partial wave}

The possible experimental observation of a spin-exotic $1^{-+} 1^{+} \rho \pi P$ wave, i.e. a partial wave with quantum numbers that cannot be construct within the framework of the quark model, is a disputed topic since decades. The results of various previous analyses differ and lead to no final conclusions. In order to get new insights into the underlying production processes, the PWA of the charged channel was performed in bins of $t^{\prime}$, which gives a detailed information about the strength of this wave in different kinematical regions. In addition the contribution of the Deck mechanism, a non-resonant process which leads to the same three-pion final state, is investigated. In the Deck process the incoming beam pion gets excited to a two-pion intermediate state and a virtual pion, which scatters diffractively from the target proton and thereby becoming real. In order to understand the influence of Deck to the obtained data, a Monte Carlo data set, based on the Deck mechanism (c.f. Eq. (4), [5]) is generated and the same PWA is applied to it:

$$
\Psi\left(m_{\pi \pi}, t_{\pi}, t\right)=\frac{A_{\pi \pi}\left(m_{\pi \pi}, t_{\pi}\right) A_{\pi p}(s, t)}{m_{\pi}^{2}-t_{\pi}}
$$


MESON 2014 $-13^{\text {th }}$ International Workshop on Production, Properties and Interaction of Mesons

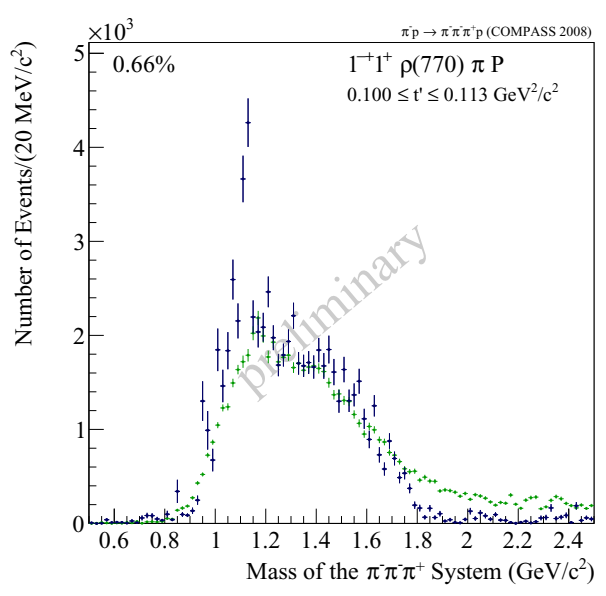

(a)

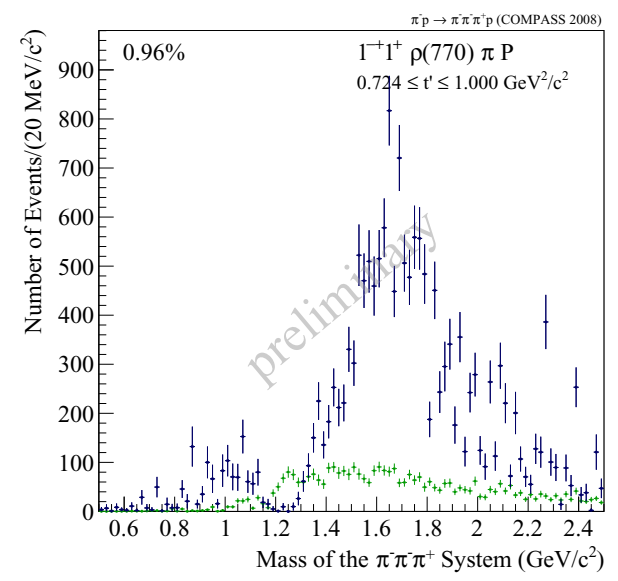

(b)

Figure 3: Intensity of the $1^{-+} 1^{+} \rho \pi P$ wave in the low $t^{\prime}$ region (a) and at high $t^{\prime}(\mathrm{b})$; real data in blue, corresponding Deck MC overlaid in green.

The obtained intensity of the Deck MC in the exotic wave is overlaid with the fit result of the real data in Fig. 3 for different kinematical regions of $t^{\prime}$. Figure 3a shows that the obtained Deck intensity (green) describes the observed intensity of the real data (blue) well at low $t^{\prime}$, while at high $t^{\prime}$ the structure in the real data around $1.6 \mathrm{GeV} / c^{2}$ cannot be described by the overlaid Deck MC intensity (c.f. Fig. 3b).

\section{Tests of chiral dynamics}

Lowering the kinematical region of $t^{\prime}$ from $0.1 \mathrm{GeV}^{2} / c^{2}$ to below $0.001 \mathrm{GeV}^{2} / c^{2}$ offers the possibility for analyses which can probe predictions of chiral perturbation theory $(\chi \mathrm{PT})$. One interesting concept is the measurement of the pion polarizability by embedding the pion-photon scattering into the Primakoff Compton reaction. A tiny extrapolation brings the virtual photon of the Primakoff reaction on shell which turns this process into a pion-photon scattering reaction, accesibble by the COMPASS spectrometer (c.f. Eq. (5)):

$$
\gamma^{(*)} \pi \rightarrow \gamma \pi
$$

Two-loop $\chi$ PT calculations predict for $\alpha_{\pi}-\beta_{\pi}, \alpha_{\pi}$ the electric and $\beta_{\pi}$ the magnetic polarizability, a value of $(5.7 \pm 1.0) \times 10^{-4} \mathrm{fm}^{3}$, under the assumption that $\alpha_{\pi}=-\beta_{\pi}$. This calculation differs significantly from previous experimental observations, which vary between 4 and 14 .

The concept of the COMPASS analysis is to compare data sets, taken with a muon and a pion beam, with Monte Carlo data, based on a pointlike beam particle. Challenging for this analysis is the precise control of the systematics. It is the goal of this analysis to obatin a value for $\alpha_{\pi}$ under the previous mentioned assumption. Fig. 4 shows the ratio real data and Monte Carlo data. The muon data are in good agreement with the Monte Carlo (c.f. Fig. 4a). In contrast to this Fig. 4b shows a difference in the compariosn of the pion data with the Monte Carlo which is caused by the pion polarizability. The obtained value for $\alpha_{\pi}$ is $\left(2.0 \pm 0.6_{\text {stat. }} \pm 0.7_{\text {sys. }}\right) \times 10^{-4} \mathrm{fm}^{3}$, which is in a good agreement with the $\chi \mathrm{PT}$ calculations. 


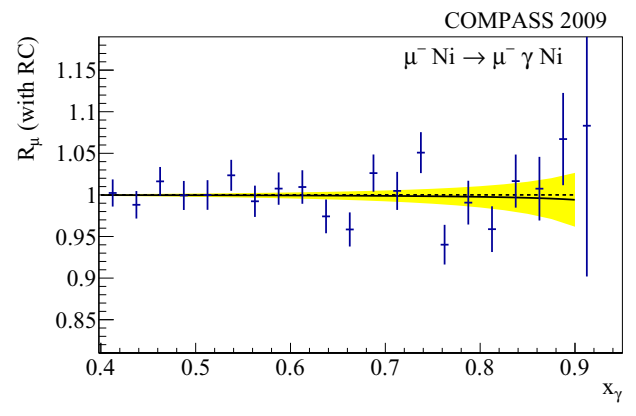

(a)

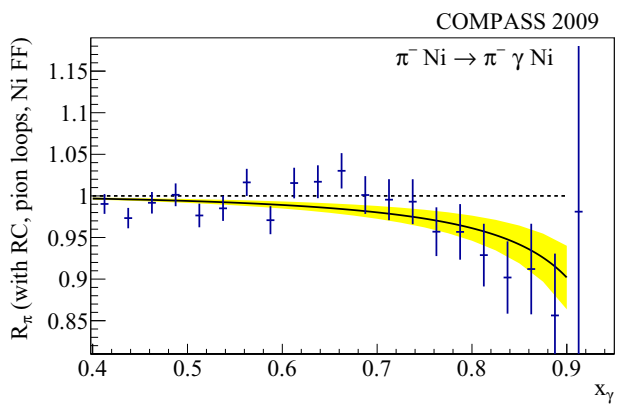

(b)

Figure 4: Ratio of real data and MC cross section for pointlike particle: muon beam data (a), pion beam data (b).

\section{Conclusions}

During the hadron structure and spectroscopy campaign in 2008 and 2009 large data sets could be obtained with the COMPASS spectrometer. Up to two orders of magnitude statistical improvement in comparison to previous analyses could be achieved for some final states like the $\pi^{-} \pi^{+} \pi^{-}$system. The physics program of COMPASS ranges from meson spectroscopy, in particular the analysis of diffractively produced $3 \pi, 5 \pi, K^{-} \pi^{+} \pi^{-}$and $\eta^{\left({ }^{\prime}\right)}$ systems, centrally produced and isobaric $2 \pi$ subsystems up to baryon spectroscopy with a proton beam focusing on $p_{f} \pi^{+} \pi^{-} p_{s}$ or $p_{f} K^{-} K^{+} p_{s}$ systems.

Another milestone of this physics program are various test of the chiral dynamics, especially the measurement of radiative widths of three-pion resonances in the kinematical intersection region of strong and Coulomb interaction and finally the measurement of the pion polarisability using the Primakoff effect.

\section{Acknowledgements}

Supported by BMBF, MLL and the Cluster of Excellence Exc153 "Origin and Structure of the Universe"

\section{References}

[1] Ph. Abbon et al. (COMPASS collaboration), (sub. Nucl. Instr. and Meth. A) CERN Preprint, 1-91 (2014).

[2] C. Adolph et al. (COMPASS collaboration), Nucl. Phys. B 886, 1078 (2014).

[3] C. Adolph et al. (COMPASS collaboration), EPJ A 50, 79 (2014).

[4] C. Adolph et al. (COMPASS collaboration), (sub. Phys. Lett. B) CERN Preprint, 1-15 (2014).

[5] C. Daum et al. (ACCMOR collaboration), Nucl. Phys. B 182, 269-336 (1981). 